\title{
Network pharmacology-based prediction of the multi-target capabilities of the compounds in Taohong Siwu decoction, and their application in osteoarthritis
}

\author{
CHUN-SONG ZHENG ${ }^{1,2}$, XIAO-JIE XU ${ }^{1,3}$, HONG-ZHI YE ${ }^{1,2}$, GUANG-WEN WU ${ }^{2}$, \\ XI-HAI LI ${ }^{2}$, HUI-FENG XU ${ }^{2}$ and XIAN-XIANG LIU ${ }^{1}$ \\ ${ }^{1}$ Fujian Academy of Integrative Medicine and ${ }^{2}$ Fujian Key Laboratory of Integrative Medicine on Geriatrics, \\ Fujian University of Traditional Chinese Medicine, Fuzhou, Fujian 350122; \\ ${ }^{3}$ College of Chemistry and Molecular Engineering, Peking University, Beijing 100871, P.R. China \\ Received February 6, 2013; Accepted May 1, 2013
}

DOI: $10.3892 /$ etm.2013.1106

\begin{abstract}
Taohong Siwu decoction (THSWD), a formulation prescribed in traditional Chinese medicine (TCM), has been widely used in the treatment of osteoarthritis (OA). TCM has the potential to prevent diseases, such as OA, in an integrative and holistic manner. However, the system-level characterization of the drug-target interactions of THSWD has not been elucidated. In the present study, we constructed a novel modeling system, by integrating chemical space, virtual screening and network pharmacology, to investigate the molecular mechanism of action of THSWD. The chemical distribution of the ligand database and the potential compound prediction demonstrated that THSWD, as a natural combinatorial chemical library, comprises abundant drug-like and lead-like compounds that may act as potential inhibitors for a number of important target proteins associated with OA. Moreover, the results of the 'compound-target network' analysis demonstrated that 19 compounds within THSWD were correlated with more than one target, whilst the maximum degree of correlation for the compounds was seven. Furthermore, the 'target-disease network' indicated
\end{abstract}

Correspondence to: Dr Xian-Xiang Liu, Fujian Academy of Integrative Medicine, Fujian University of Traditional Chinese Medicine, 1 Huatuo Road, Minhou Shangjie, Fuzhou, Fujian 350122, P.R. China

E-mail: liuxianxiang@163.com

Abbreviations: THSWD, Taohong Siwu decoction; TCM, traditional Chinese medicine; ADAMTS-4, aggrecanase-1; MMP, matrix metalloproteinase; TNF- $\alpha$, tumor necrosis factor- $\alpha$; iNOS, inducible nitric oxide synthase; COX, cyclooxygenase; CDK, cyclin-dependent kinase; HO, heme oxygenase; PPAR $\gamma$, peroxisome proliferator-activated receptor- $\gamma$; VDR, vitamin D nuclear receptor; TTD, therapeutic target database

Key words: Taohong Siwu decoction, osteoarthritis, multi-target, network pharmacology that THSWD may potentially be effective against 69 diseases. These results may aid in the understanding of the use of THSWD as a multi-target therapy in OA. Moreover, they may be useful in establishing other pharmacological effects that may be brought about by THSWD. The in silico method used in this study has the potential to advance the understanding of the molecular mechanisms of TCM.

\section{Introduction}

Osteoarthritis (OA) is a disease that is induced through several complex mechanisms, including the progressive erosion of articular cartilage, proteoglycan (PG) degradation and the disruption of the collagen network. It is the most common form of arthritis, and is a major cause of morbidity, limitations in activity, physical disability, excessive health care utilization and a reduced health-related quality of life, particularly in individuals aged $\geq 45$ years (1). Treatments for OA are widely perceived as an unmet medical requirement. Until recently, there has been no specific remedy for the disease, despite the availability of supplements, such as chondroitin sulfate, and the wide range of effective analgesics and non-steroidal anti-inflammatory drugs (NSAIDs) that are used as treatments for OA. However, the pharmacological management of OA has targeted the symptoms of the disease, rather than the underlying causes $(2,3)$. Thus, there have been efforts to develop drugs that reverse, retard or stabilize the underlying pathological changes in $\mathrm{OA}$, and thereby provide a long-term symptomatic relief.

At present, efforts are being focused on the discovery of drugs that affect multiple targets and levels of the disease network. A diverse range of studies have been performed in the emerging field of multi-target drug design (4-7). In addition, several studies have demonstrated that an improved method of treating OA may be to act on several targets simultaneously $(6,8)$. It has also been indicated that the substances used in traditional Chinese medicine (TCM) possess diverse biological activities, and their composition and pharmacodynamic action are improved when administered in conjunction, as opposed to individually (9). Therefore, the establishment 
of a comprehensive method that elucidates the multi-target capabilities of TCM is required for the development of new drugs to treat OA.

Taohong Siwu decoction (THSWD) is a TCM that was documented in 'YiZong JinJian' (compiled by Wu Qian in the Qing Dynasty), and has been prescribed to treat OA in China. It has been demonstrated that THSWD effectively inhibits the expression of matrix metalloproteinase (MMP)-1 in the articular cartilage of rats with knee osteoarthritis (KOA), modeled by Hulth's method, and reduces the levels of interleukin (IL)-1 and IL-6 in the localized tissues of the $\operatorname{KOA}(10,11)$. However, the underlying mechanisms of THSWD in the management of OA are poorly understood. With the progress that has been made in systems biology and bionetworks, network pharmacology has been used to study the functions of herbal formulations from a proteome or systematic level (12). Therefore, the aim of the present study was to elucidate the multi-target capabilities of the compounds in THSWD, based on the established methods of network pharmacology $(13,14)$. In addition, the study aimed to promote the understanding of the efficiency of THSWD, with regard to its application in OA, and to facilitate its modernization and global use.

\section{Materials and methods}

Construction and preparation of the compound ligand database. THSWD consists of six species of medicinal herbs: Radix Rehmanniae Preparata (Shudihuang), Angelica sinensis (Danggui), Radix Paeoniae Alba (Baishao), Ligusticum chuanxiong (Chuanxiong), Prunus persica (Taoren) and Carthamus annuum (Honghua). The compounds identified in the medicinal herbs of THSWD were determined from the Chinese Herbal Drug Database and the Handbook of the Chemical Constituents in Chinese Herb Original Plants $(15,16)$. Having excluded any duplicates, the total number of compounds was 206. The structures of the compounds were drawn using ISIS Draw, Version 2.5 (MDL Information Systems, Inc., San Leandro, CA, USA), and further optimized using Discovery Studio 2.0 (DS 2.0; Accelrys, Inc., San Diego, CA, USA), with a Merck molecular force field (MMFF).

The molecular descriptors of the ligand database were calculated in the quantitative structure-activity relationship (QSAR) module of DS 2.0 (Accelrys, Inc.), whilst the chemical space of the ligand database was constructed using 150 diversity descriptors, including 1D, 2D and 3D molecular descriptors. Principal component analysis (PCA) was then performed to map the chemical distribution of the ligands in chemical space.

Virtual docking screening. To highlight the THSWD components that were particularly likely to be active in the OA disease system, a docking protocol was performed, in order to identify any interactions with the common OA target enzymes. This was completed using DS 2.0 LigandFit (Accelrys, Inc.) (7).

The $3 \mathrm{D}$ crystal structures of the 15 protein targets associated with OA, as determined by the Therapeutic Targets Database (TTD; Bioinformatics and Drug Design group, National University of Singapore) and other literature sources (17-20), were obtained from the Research Collabatory for Structural Bioinformatics (RCSB) Protein Data Bank (PDB; Table I), and
Table I. Fifteen key protein targets associated with osteoarthritis.

\begin{tabular}{lc}
\hline Protein & PDB code \\
\hline ADAMTS-4 & $2 \mathrm{RJP}$ \\
TNF- $\alpha$ & $2 \mathrm{AZ5}$ \\
iNOS & $2 \mathrm{Y} 37$ \\
COX-1 & $3 \mathrm{NT} 1$ \\
COX-2 & $3 \mathrm{~N} 8 \mathrm{X}$ \\
MMP-1 & 966C \\
MMP-3 & $1 \mathrm{C} 3 \mathrm{I}$ \\
MMP-8 & $1 \mathrm{ZS} 0$ \\
MMP-9 & $1 \mathrm{GKC}$ \\
MMP-12 & $3 \mathrm{RTS}$ \\
MMP-13 & $3 \mathrm{I} 7 \mathrm{I}$ \\
VDR & $1 \mathrm{DB} 1$ \\
PPAR $\gamma$ & $2 \mathrm{VSR}$ \\
CDK2 & $3 \mathrm{PXY}$ \\
HO-1 & 3TGM
\end{tabular}

PDB, Protein Data Bank; ADAMTS-4, aggrecanase-1; TNF- $\alpha$, tumor necrosis factor- $\alpha$; iNOS, inducible nitric oxide synthase; COX, cyclooxygenase; MMP, matrix metalloproteinase; VDR, vitamin D nuclear receptor; PPAR $\gamma$, peroxisome proliferator activated receptor- $\gamma$, CDK2, cyclin-dependent kinase-2; HO-1, heme oxygenase.

uploaded into DS 2.0 (Accelrys, Inc.). The crystallographic water was removed, and hydrogen atoms were added. The inhibitor from the PDB file was used to define the active site, and the 206 compounds identified in THSWD were docked into the protein models. The ligand position and orientation were evaluated using DockScores, on the basis of the most favorable energy interactions between the ligand conformations and receptor proteins, as described previously (21). The 206 docked structures were thus sorted according to their DockScores.

Network construction and analysis. Three networks were constructed for the purpose of the study. To create a candidate compound-candidate target (cC-cT) network, $242 \mathrm{drug} / \mathrm{drug}$-like compounds associated with the 15 previously mentioned protein targets, were obtained from the TTD (17). Known compounds and their targets were used to generate a bipartite graph of compound-protein interactions, in which a compound and a protein were connected to each other if the protein was an action target of the compound. This gave rise to the cC-cT network. To create a potential compound-potential target ( $\mathrm{pC}-\mathrm{pT})$ network, the top five of the DockScore-sorted THSWD compounds were selected as potential compounds (18). The pC-pT network was established by connecting the potential compounds to any corresponding potential targets that were associated with OA. The target-disease (T-D) network was constructed by connecting the 15 previously mentioned proteins to any associated diseases, as established from the TTD. Cytoscape 2.8.3 (Cytoscape Consortium; http://www.cytoscape.org/) was used in the construction of all the networks, as described previously (22). The compounds, targets and diseases were represented as nodes in the networks. The edges between 
Table II. Mean, standard deviation, minimum and maximum values for the key variables of the compounds in Taohong Siwu decoction.

\begin{tabular}{lrrrr}
\hline Variable & Mean & SD & Minimum & Maximum \\
\hline Molecular weight & 321.59 & 180.74 & 59.11 & 938.66 \\
No. of hydrogen acceptors & 5.97 & 5.64 & 0.00 & 26.00 \\
No. of hydrogen donors & 3.19 & 3.60 & 0.00 & 17.00 \\
ALogP & 1.71 & 3.62 & -9.55 & 13.60 \\
Molecular volume & 219.39 & 111.99 & 53.16 & 9.00 \\
Wiener index & 2179.23 & 3322.42 & 12.00 & 18291.00 \\
Zagreb index & 115.26 & 71.47 & 368.00 \\
\hline
\end{tabular}

$\mathrm{SD}$, standard deviation.

Table III. Simple parameters of the (cC-cT) and (pC-pT) networks.

\begin{tabular}{lcc}
\hline Simple parameters & pC-pT network & cC-cT network \\
\hline Connected components & 1 & 6 \\
Network density & 0.054 & 0.010 \\
Network heterogeneity & 0.636 & 2.976 \\
Network centralization & 0.083 & 0.382 \\
Characteristic path length & 4.576 & 3.800 \\
Average no. neighbors & 2.830 & 2.488 \\
Shortest paths & $2756(100 \%)$ & $47636(71 \%)$ \\
\hline
\end{tabular}

pC-pT, potential compound-potential target; cC-cT, candidate compound-candidate target.

A

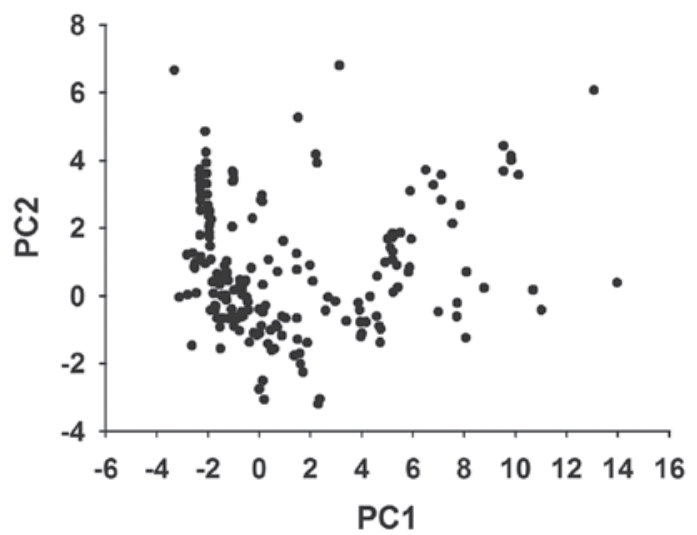

B

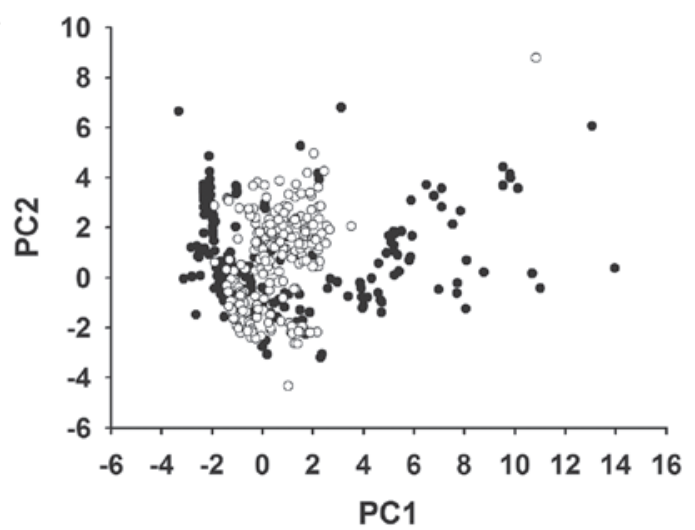

Figure 1. Global property map of the chemical space of compounds. The black circles represent the compounds retrieved from Taohong Siwu decoction, while the white circles represent the compounds retrieved from the Therapeutic Targets Database (TTD; Bioinformatics and Drug Design group, National University of Singapore). PC1, first principal component; PC2,second principal component.

the nodes represented intermolecular interactions. Data were analyzed using Cytoscape plugins.

\section{Results}

Chemical distribution of the ligand database. The global property map of the chemical space of the compounds in THSWD is depicted in Fig. 1A, which demonstrates that the compounds possess a broad diversity in chemical space. In addition, several key molecular descriptors were statistically analyzed, and are listed in Table II. These results indicated that the majority of the THSWD compounds had desired drug-like properties, according to Lipinski's rule of five. To further confirm the drug-like properties of the THSWD compounds, the chemical space distribution of the known drug/drug-like compounds associated with the OA targets, as established 


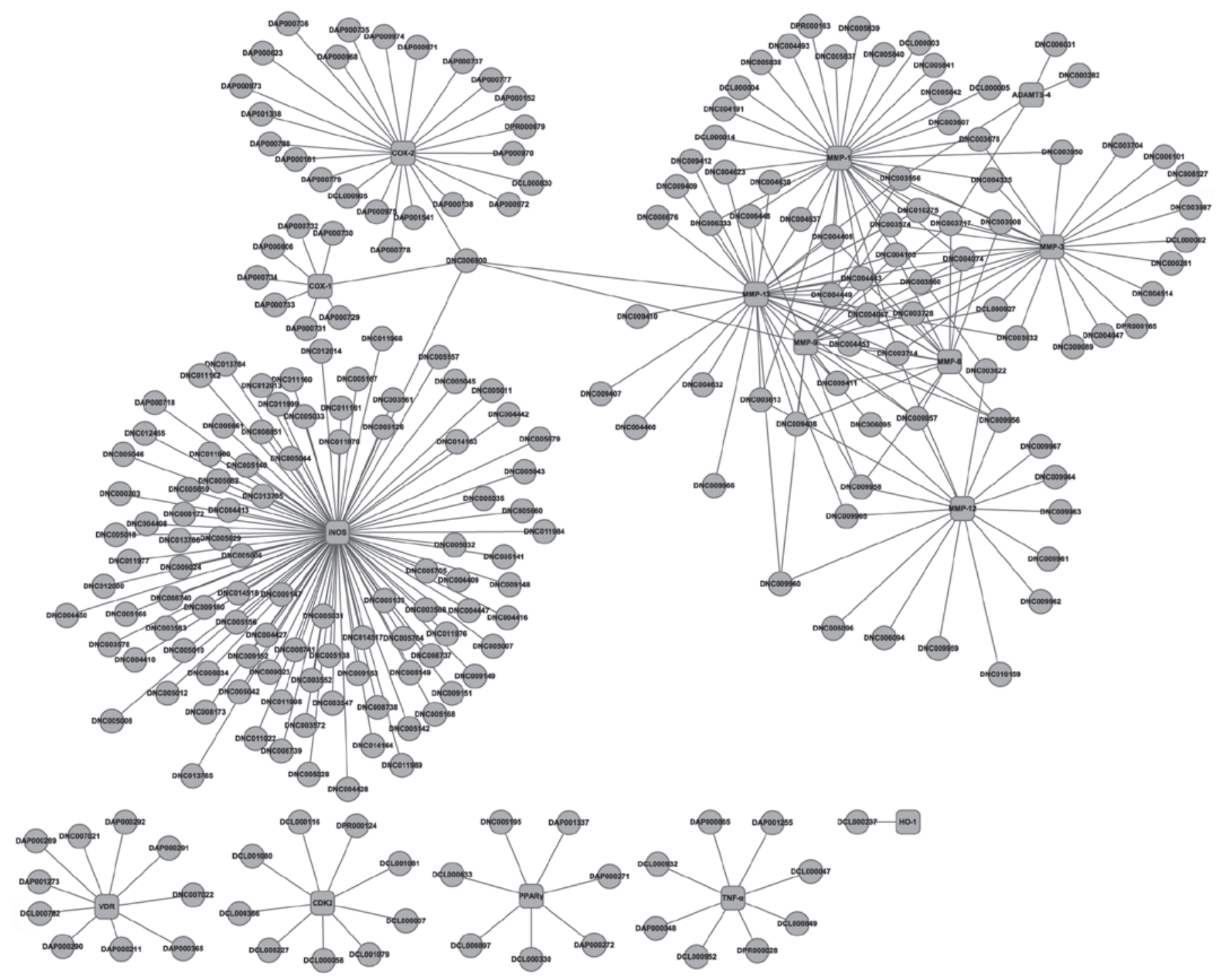

Figure 2. Candidate compound-candidate target (cC-cT) network. The circles and rounded rectangles represent the candidate compounds and target proteins, respectively.

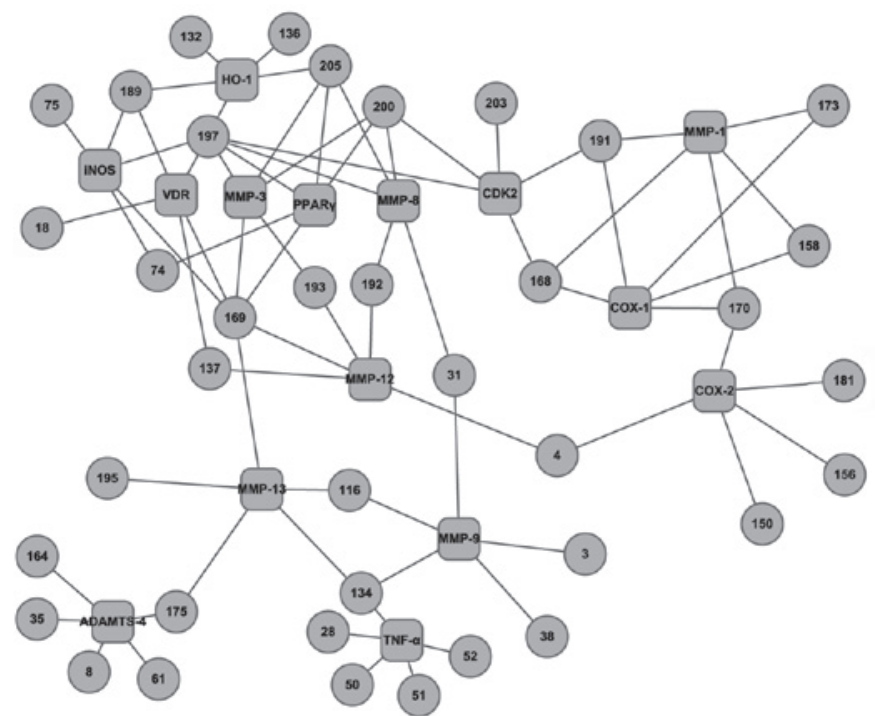

Figure 3. Potential compound-potential target (pC-pT) network. The circles and rounded rectangles represent the potential compounds and target proteins, respectively. from the TTD, were also charted. It was revealed that there were marked overlaps between these two molecular datasets in chemical space (Fig. 1B), which indicated that THSWD contained drug-like and lead-like compounds.

Potential compound prediction. The potential compound prediction was performed for the 15 proteins associated with OA using virtual screening. Docking revealed that the total number of potential compounds that exhibited desired interactions with the proteins was 38 . Among these 38 compounds, 19 demonstrated potential biological activity with more than one target protein. These results indicated that THSWD contained a number of active compounds, which had the potential to lead to the broad-spectrum inhibition of a number of important target proteins.

C-T network construction and analysis results. The $\mathrm{cC}-\mathrm{cT}$ and pC-pT networks are demonstrated in Figs. 2 and 3, respectively. A histogram of the distribution of the number of targets correlated with each compound, and corresponding with the cC-cT 
Table IV. Sixty-nine diseases correlated with the 15 potential target proteins.

Index Disease

\begin{tabular}{|c|c|}
\hline D1 & Abdominal aortic aneurysm \\
\hline D2 & Acute lymphoblastic leukemia (ALL) \\
\hline D3 & Acute myeloid leukemia (AML) \\
\hline D4 & Adenomatous polyposis \\
\hline D5 & ACTH-secreting pituitary tumors \\
\hline D6 & Advanced lung cancer \\
\hline D7 & Advanced solid tumors \\
\hline D8 & Alzheimer's disease \\
\hline D9 & Arthritis \\
\hline D10 & Asthma \\
\hline D11 & Atherosclerosis \\
\hline D12 & Autoimmune diseases \\
\hline D13 & B-cell malignancies \\
\hline D14 & Behcet's disease \\
\hline D15 & Bladder cancer \\
\hline D16 & Brain cancer \\
\hline D17 & Breast cancer \\
\hline D18 & Carcinoma in situ, unspecified \\
\hline D19 & Carpal tunnel syndrome \\
\hline D20 & Chondrosarcoma \\
\hline D21 & Chronic lymphocytic leukemia (CLL) \\
\hline D22 & Chronic myeloid leukemia \\
\hline D23 & Colorectal cancer \\
\hline D24 & Crohn's disease \\
\hline D25 & Diabetes mellitus \\
\hline D26 & Dysmenorrhea, unspecified \\
\hline D27 & Emphysema \\
\hline D28 & Endometriosis \\
\hline D29 & Gastro-intestinal ulcers \\
\hline D30 & Genitourinary tumors \\
\hline D31 & Gestational hypertension \\
\hline D32 & Guillain-Barré syndrome \\
\hline D33 & Heart failure \\
\hline D34 & Hepatocellular carcinoma \\
\hline D35 & Hormone-refractory prostate cancer \\
\hline D36 & Hyperimmunoglobulinemia D \\
\hline D37 & Inflammation \\
\hline D38 & Inflammatory bowel disease \\
\hline D39 & Insulin resistance \\
\hline D40 & Ischemia reperfusion injuries \\
\hline D41 & Ischemic heart disease \\
\hline D42 & Kaposi's sarcoma \\
\hline D43 & Lung cancer \\
\hline D44 & Meningioma \\
\hline D45 & Multiple sclerosis \\
\hline D46 & Myocardial infarction \\
\hline D47 & Nasopharyngeal cancer (NPC) \\
\hline D48 & Neonatal hyperbilirubinemia, jaundice \\
\hline D49 & Non-Hodgkin's lymphoma \\
\hline D50 & Noninsulin-dependent diabetes mellitus \\
\hline D51 & Non-small cell lung cancer \\
\hline
\end{tabular}

Table IV. Continued.

\begin{tabular}{ll}
\hline Index & Disease \\
\hline D52 & Obesity \\
D53 & Osteoarthritis \\
D54 & Osteoporosis, unspecified \\
D55 & Ovarian cancer \\
D56 & Pain, unspecified \\
D57 & Pancreatic cancer \\
D58 & Pathological angiogenesis \\
D59 & Peutz-Jeghers syndrome \\
D60 & Prostate cancer \\
D61 & Psoriasis \\
D62 & Renal cell carcinoma \\
D63 & Rheumatic diseases \\
D64 & Rheumatoid arthritis, unspecified \\
D65 & Squamous cell carcinoma \\
D66 & Stroke \\
D67 & Testicular cancer \\
D68 & Thyroid follicular carcinoma \\
D69 & Ulcerative colitis \\
\hline
\end{tabular}

ACTH, Adrenocorticotrophic hormone.

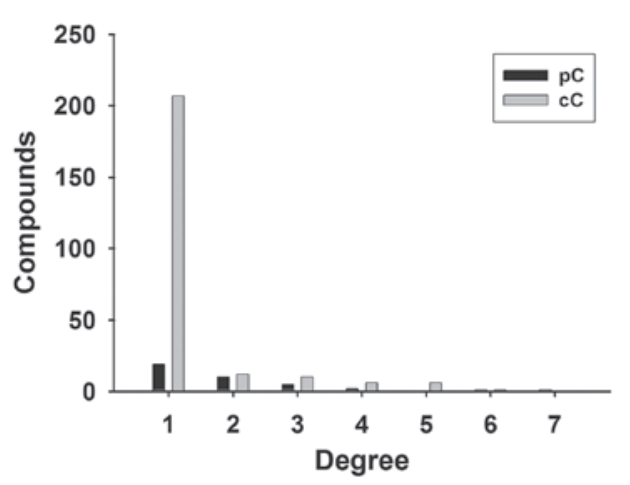

Figure 4. Distribution of the number of targets associated with each compound. pC, potential compound; $\mathrm{cC}$, candidate compound.

and $\mathrm{pC}$-pT networks, is demonstrated in Fig. 4. The simple parameters of the cC-cT and pC-pT networks are exhibited in Table III. These results indicated that the majority of the candidate and potential compounds interacted with only one target protein; however, certain compounds were identified to be modulators of multiple targets.

T-D network construction and analysis results. A total of 15 validated potential targets and the 69 diseases that were associated with them, as established from the TTD website, were used to construct the T-D network (Table IV and Fig. 5). According to the US National National Library's Medical Subject Headings (http://www.nlm.nih.gov/mesh), the 69 diseases were classified into 20 groups. For example, OA, osteoporosis and rheumatoid arthritis were grouped as musculoskeletal diseases, while heart failure, myocardial infarction 


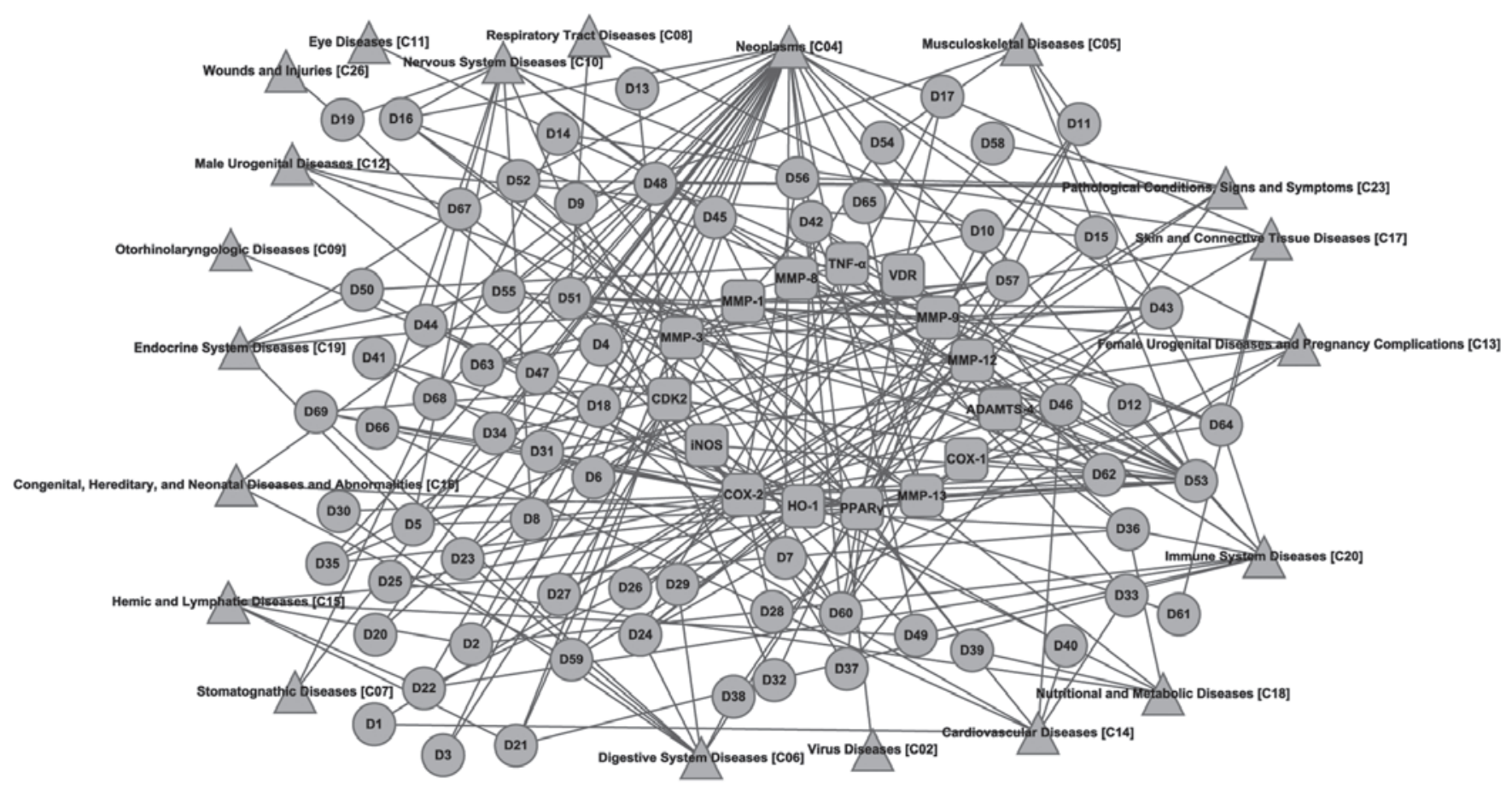

Figure 5. Target-disease network of 15 potential targets (rounded rectangles) connected to 69 diseases (circles), which were classified into 20 groups (triangles) according to the US National Library of Medicine's Medical Subject Headings (http://www.nlm.nih.gov/mesh).

and ischemia reperfusion injuries were classified as cardiovascular diseases. This suggests that THSWD may demonstrate efficacy in targeting not only musculoskeletal diseases, but also neoplasms, nervous system diseases and cardiovascular diseases, amongst others.

\section{Discussion}

The chemical space of the compounds in THSWD was defined by calculating a given set of descriptors for each molecule, and by using these values as coordinates in the multidimensional space. PCA was performed to map the multiple descriptor values into a $2 \mathrm{D}$ plane. This provided a novel method for demonstrating the established theory among chemists that similar compounds have similar properties (23). Fig. 1A reveals that the compounds in THSWD possess a broad diversity in chemical space, which indicates that THSWD, as a natural combinatorial chemical library, has the potential to exhibit the desired interactions with a wider range of protein targets than the drug/drug-like compounds from the TTD. The marked overlap in Fig. 1B suggests that THSWD is likely to contain active compounds that have the potential to exert synergistic therapeutic actions. The statistics of the drug-like properties listed in Table II show that the means of the molecular weight, the number of hydrogen bond donors, the number of hydrogen bond acceptors and AlogP are 321.59, 3.19, 5.97 and 1.71, respectively. According to Lipinski's rule of five (24), these results suggest that the majority of the compounds in THSWD have desired drug-like properties, and are suitable for screening lead compounds.

Using virtual docking screening, we demonstrated that THSWD contains certain potential promiscuous and combination drugs (50\% for each); therefore, multiple active compounds in THSWD may simultaneously target several proteins associated with OA. In order to enhance our understanding of the functions of THSWD, the property profile of the potential compound-target interactions was compared with that of the candidate compound-target interactions, established from the TTD. The cC-cT network was generated by six connected components, with the candidate compounds in the outer circle of each component demonstrating fewer interactions with the target proteins. The mean number of candidate targets per candidate compound was 1.3 . However, the pC-pT network consisted of 53 nodes ( 38 potential compounds and 15 potential targets) and 75 edges. The mean number of potential targets per potential compound was 2.0. Among the 38 potential compounds, nine were capable of acting on more than two targets. These potential compounds included 6-hydroxykaempferol-7-O-glucoside, folic acid, neocarthamin, safflor yellow A, ferulic acid, eugeniin, (Z)-5-hydroxy-3-butylidene-phthalide, carthamidin and amygdalin. Previous studies have revealed that the majority of these compounds exhibit biological activity against OA (25-28). A maximum of seven targets were correlated with one potential compound. Notably, the pC-pT network contained only one component, while the cC-cT network contained six. This indicated that the anti-OA action of THSWD was a result of holistic combinations attacking different targets, rather than a precise attack on a single target. Therapeutic polypharmacology involves the theory of treating multigenic, complex diseases by targeting multiple targets with one or more drugs, in order to effectively reset the regulatory network processes that are altered in the disease state (29). Therefore, therapeutic polypharmacology may explain why THSWD is effective in the treatment of OA.

In order to further understand the therapeutic polypharmacology of THSWD, a T-D network was contructed to study 
the correlation between the diseases and targets. As demonstrated in Fig. 5, the majority of the diseases were classified as neoplasms and nervous system and cardiovascular diseases, in addition to musculoskeletal diseases. This suggests that THSWD is capable of exerting broad pharmacological effects. Amygdalin, for example, was indicated as a potential compound in THSWD. Amygdalin may treat OA by targeting heme oxygenase (HO)-1, an enzyme that has been associated with cardiovascular and musculoskeletal diseases, as well as neoplasms. In addition, it has been demonstrated that amygdalin has exhibited therapeutic potential in the prevention and treatment of atherosclerosis (30). Furthermore. amygdalin may significantly reduce plasma viscosity, prolong thromboplastin time and reduce fibrinogen levels, whilst exerting few effects on whole blood viscosity (31). Amygdalin has also been used for the treatment of asthma, tumors, bronchitis, emphysema, leprosy and diabetes (32). Clinical observations have indicated the efficacy of THSWD in internal medicine, gynecology, andriatrics, dermatology and ophthalmology (33). Therefore, taking into account the multi-component and multi-target patterns of THSWD, it is possible that THSWD may improve the efficiency of treatments for other diseases, aside from OA. It would be useful to determine the potential pharmacological effects of THSWD, in order to aid the development of new and effective therapeutic drugs from THSWD.

In conclusion, we have constructed a novel modeling system, by integrating chemical space, virtual screening and network pharmacology, to investigate the molecular mechanism of action of THSWD, with regard to its clinical efficacy against OA. Our results demonstrated that THSWD has an improved structural diversity, compared with the selected drug/drug-like compounds from the TTD, and contains several active compounds capable of targeting multiple OA-related proteins. Furthermore, the $\mathrm{cC}-\mathrm{cT}$ and $\mathrm{pC}$-pT networks revealed that THSWD possesses the properties of promiscuous and combination drugs for multi-target OA therapies. Moreover, the T-D network indicates that THSWD had therapeutic potential against diseases other than $\mathrm{OA}$, such as cardiovascular diseases and neoplasms. This suggests that distinct diseases may be treated with the same TCM formula. As a whole, these results indicate that TCM formulations contain compounds with multi-target capabilities and broad pharmacological effects.

\section{Acknowledgements}

This study was supported by the Developmental Fund of Chen Keji Integrative Medicine (grant no. CKJ2010032) and the National Natural Science Foundation of China (grant no. 81202713).

\section{References}

1. Helmick CG, Felson DT, Lawrence RC, Gabriel S, Hirsch R, Kwoh CK, Liang MH, Kremers HM, Mayes MD, Merkel PA, et al; National Arthritis Data Workgroup: Estimates of the prevalence of arthritis and other rheumatic conditions in the United States. Part I. Arthritis Rheum 58: 15-25, 2008.

2. Choi JH, Choi JH, Kim DY, Yoon JH, Youn HY, Yi JB, Rhee HI, Ryu KH, Jung K, Han CK, et al: Effects of SKI 306X, a new herbal agent, on proteoglycan degradation in cartilage explant culture and collagenase-induced rabbit osteoarthritis model. Osteoarthritis Cartilage 10: 471-478, 2002.
3. Hochberg MC: Osteoarthritis year 2012 in review: clinical. Osteoarthritis Cartilage 20: 1465-1469, 2012.

4. Zimmermann GR, Lehár J and Keith CT: Multi-target therapeutics: when the whole is greater than the sum of the parts. Drug Discov Today 12: 34-42, 2007.

5. Yang K, Bai H, Ouyang Q, Lai L and Tang C: Finding multiple target optimal intervention in disease-related molecular network. Mol Syst Biol 4: 228, 2008.

6. Frantz S: Drug discovery: playing dirty. Nature 437: 942-943, 2005.

7. Zheng CS, Xu XJ and Ye HZ: Computational simulation of multi-target research on the material basis of Caulis sinomenii in treating osteoarthritis. Zhongguo Zhong Xi Yi Jie He Za Zhi 32: 375-379, 2012 (In Chinese).

8. Wu SQ, Otero M, Unger FM, Goldring MB, Phrutivorapongkul A, Chiari C, Kolb A, Viernstein H and Toegel S: Anti-inflammatory activity of an ethanolic Caesalpinia sappan extract in human chondrocytes and macrophages. J Ethnopharmacol 138: 364-372, 2011.

9. Dou SS, Liu RH, Jiang P, Liu L, Zhang C, Luo GA and Zhang WD: System biology and its application in compound recipe of traditional Chinese medicine study. World Science and Technology 10: 116-121, 2008.

10. Li N, Song M, Song ZJ and Xie XW: The effects of modified Taohong Siwu Tang on MMP-1 and TIMP-1 in articular cartilage of the rats with KOA. Journal of Gansu College of Traditional Chinese Medicine 26(6): 12-14, 2009 (In Chinese).

11. Li N, Xie XW and Song M: Effects of Taohongsiwu decoction and Simiao decoction on the expressions of interleukin- $1 \beta$ and interleukin-6 in rat knee osteoarthritis. Journal of Lanzhou University (Medical Sciences) 35(2): 24-26, 30, 2009 (In Chinese).

12. Liu ZH and Sun XB: Network pharmacology: new opportunity for the modernization of traditional Chinese medicine. Yao Xue Xue Bao 47: 696-703, 2012 (In Chinese).

13. Gu J, Zhang H, Chen L, Xu S, Yuan G and Xu X. Drug-target network and polypharmacology studies of a Traditional Chinese Medicine for type II diabetes mellitus. Comput Biol Chem 35: 293-297, 2011.

14. Tao W, Xu X, Wang X, Li B, Wang Y, Li Y and Yang L: Network pharmacology-based prediction of the active ingredients and potential targets of Chinese herbal Radix Curcumae formula for application to cardiovascular disease. J Ethnopharmacol 145: 1-10, 2013.

15. Qiao X, Hou T, Zhang W, Guo S and Xu X: A 3D structure database of components from Chinese traditional medicinal herbs. J Chem Inf Comput Sci 42: 481-489, 2002.

16. Zhou JJ, Xie GR and Yang JX (eds): Handbook of the Chemical Constituents in Chinese Herb Original Plants. Chemical Industry Press, Beijing, pp1165-1211, 2004 (In Chinese).

17. Zhu F, Shi Z, Qin C, Tao L, Liu X, Xu F, Zhang L, Song Y, Liu X, Zhang J, Han B, Zhang P and Chen Y: Therapeutic target database update 2012: a resource for facilitating target-oriented drug discovery. Nucleic Acids Res 40: D1128-D1136, 2012.

18. Zheng CS, Ye HZ, Cai LL, Chen JS, Wei LS and Liu XX: Discussion on multi-component and multi-target pattern of Liuweidihuang pill for osteoarthritis based on computer simulation. Zhongyi Zhenggu 25: 11-18, 2013 (In Chinese)

19. Chang CC, Hsieh MS, Liao ST, Chen YH, Cheng CW, Huang PT, Lin YF and Chen CH: Hyaluronan regulates PPAR $\gamma$ and inflammatory responses in IL-1 $\beta$-stimulated human chondrosarcoma cells, a model for osteoarthritis. Carbohydr Polym 90: 1168-1175, 2012.

20. Liu YP and Yu GR: Comment on 'the matrix metalloproteinases as pharmacological target in osteoarthritis: statins may be of therapeutic benefit'. Med Hypotheses 74: 394-395, 2010.

21. Montes M, Braud E, Miteva MA, Goddard ML, Mondésert O, Kolb S, Brun MP, Ducommun B, Garbay C and Villoutreix BO: Receptor-based virtual ligand screening for the identification of novel CDC25 phosphatase inhibitors. J Chem Inf Model 48: $157-165,2008$

22. Smoot ME, Ono K, Ruscheinski J, Wang PL and Ideker T: Cytoscape 2.8: new features for data integration and network visualization. Bioinformatics 27: 431-432, 2011.

23. Dobson CM: Chemical space and biology. Nature 432: $824-828$, 2004.

24. Lipinski CA, Lombardo F, Dominy BW and Feeney PJ: Experimental and computational approaches to estimate solubility and permeability in drug discovery and development settings. Adv Drug Deliv Rev 46: 3-26, 2001 
25. Liu BX, Chen LB, Wang H, Qin J and Wang P: Effects of angelica and sodium ferulate injection on cartilage degeneration and metabolism of free radicals in rats with osteoarthritis. Medical Journal of Wuhan University 27: 626-628, 2006 (In Chinese).

26. Liu BX, Chen LB, Wang H, Qin J and Shang L: Therapeutic effects of angelica and sodium ferulate on experimental osteoarthritis in rats. Orthopedic Journal of China 16: 1316-1318, 2008.

27. Wang ZY, Ren H and Wang HM. The articular cavity washing with safflower injection combined with bearded needle debonding cure knee osteoarthritis. Proceeding of Clinical Medicine 21: 494-497, 2012 (In Chinese).

28. Li JS and Zhao LG: Research progress of amygdalin. Huaxia Medicine 2: 326-330, 2007 (In Chinese).

29. Boran AD and Iyengar R: Systems approaches to polypharmacology and drug discovery. Curr Opin Drug Discov Devel 13: 297-309, 2010.
30. Jiagang D, Li C, Wang H, Hao E, Du Z, Bao C, Lv J and Wang Y: Amygdalin mediates relieved atherosclerosis in apolipoprotein $\mathrm{E}$ deficient mice through the induction of regulatory $\mathrm{T}$ cells. Biochem Biophys Res Commun 411: 523-529, 2011.

31. Liu L, Duan JA, Tang Y, Guo J, Yang N, Ma H and Shi X: Taoren-Honghua herb pair and its main components promoting blood circulation through influencing on hemorheology, plasma coagulation and platelet aggregation. J Ethnopharmacol 139: 381-387, 2012.

32. Zhou CS, Qian LC, Ma HL, Yu XJ, Zhang YZ, Qu WJ, Zhang XX and Xia W: Enhancement of amygdalin activated with $\beta$-D-glucosidase on HepG2 cells proliferation and apoptosis. Carbohydr Polym 90: 516-523, 2012.

33. Li Y and Peng DY: Progress in pharmacological research of Taohong Siwu Decoction. Anhui Medical and Pharmaceutical Journal 15: 529-531, 2011 (In Chinese). 